\title{
Accurate and Efficient Nyström Volume Integral Equation Method for the Maxwell equations for Multiple 3-D Scatterers
}

\author{
Duan Chen ${ }^{a}$ Wei Cai ${ }^{a}$ Brian Zinser ${ }^{a}$ Min Hyung Cho ${ }^{b}$ \\ ${ }^{a}$ Department of Mathematics and Statistics, University of North Carolina at \\ Charlotte, Charlotte, NC 28223, USA \\ ${ }^{\mathrm{b}}$ Department of Mathematical Sciences, University of Massachusetts Lowell, \\ Lowell, MA 01854, USA
}

\author{
Suggested Running Head: \\ Accurate and Efficient Nyström Volume Integral Equations for \\ the Maxwell equations \\ Corresponding Author: \\ Prof. Wei Cai \\ Department of Mathematics and Statistics, \\ University of North Carolina at Charlotte, \\ Charlotte, NC 28223-0001 \\ Phone: 704-687-0628, Fax: 704-687-6415, \\ Email: wcai@uncc.edu
}




\title{
AMS Subject classifications: 65R20, 65Z05, 78M25
}

\begin{abstract}
In this paper, we develop an accurate and efficient Nyström volume integral equation (VIE) method for the Maxwell equations for large number of 3-D scatterers. The Cauchy Principal Values that arise from the VIE are computed accurately using a finite size exclusion volume together with explicit correction integrals consisting of removable singularities. Also, the hyper-singular integrals are computed using interpolated quadrature formulae with tensor-product quadrature nodes for several objects, such as cubes and spheres, that are frequently encountered in the design of meta-materials . The resulting Nyström VIE method is shown to have high accuracy with a minimum number of collocation points and demonstrate $p$-convergence for computing the electromagnetic scattering of these objects. Numerical calculations of multiple scatterers of cubic and spherical shapes validate the efficiency and accuracy of the proposed method.
\end{abstract}

Key words: Electromagnetic (EM) scattering, volume integral equation, Cauchy Principal Value, dyadic Green's function, Nyström methods.

\section{Introduction}

Electromagnetic (EM) wave scattering of random microstructures occurs in a wide range of applications. For example, the interaction of light with surface plasmons on roughened metallic surfaces produces surface plasmon polaritons (SPP) [213, which has important applications in solar cells [3], meta-materials, and super-resolution imaging devices [12,6]. Also, surface enhanced Raman scattering (SERS) [14] is closely related to the excitation of surface plasmons on rough or nano-pattern surfaces by incident light and is a very useful tool in finger-printing chemical components of a molecule, single molecule detector, DNA detection, and bio-sensor, etc [7]. In all these applications, it is critical to have accurate and efficient numerical methods for computer simulations of the EM scattering of a large number of microscopic objects such as spheres, cubes, etc.

In this paper, we will present an accurate and efficient Nyström volume integral equation (VIE) method for the time harmonic Maxwell equations using dyadic Green's functions $\overline{\mathbf{G}}_{\mathbf{E}}\left(\mathbf{r}, \mathbf{r}^{\prime}\right)$. In most applications, the scatterers are embedded in either a homogeneous or a layered medium. Thus, a VIE can be derived for the regions occupied by the scatterers using the dyadic Green's 
function $\overline{\mathbf{G}}_{\mathbf{E}}\left(\mathbf{r}, \mathbf{r}^{\prime}\right)$. The resulting VIE is a second kind Fredholm integral equation and $\overline{\mathbf{G}}_{\mathbf{E}}\left(\mathbf{r}, \mathbf{r}^{\prime}\right)$ will ensure that the scattered field, expressed in terms of equivalent current sources inside the scatterer, satisfies the Silver-Müller radiation conditions at infinity [4]. In the VIE formulation, computing the electric field inside the scatterer will involve the use of Cauchy Principal Values (CPV or simply p.v.) associated with the dyadic Green's function, namely,

$$
\text { p.v. } \int_{\Omega} \mathrm{d} \mathbf{r}^{\prime} \mathrm{i} \omega \overline{\mathbf{G}}_{\mathbf{E}}\left(\mathbf{r}, \mathbf{r}^{\prime}\right) \cdot \Delta \epsilon\left(\mathbf{r}^{\prime}\right) \mathbf{E}\left(\mathbf{r}^{\prime}\right)=\lim _{\delta \rightarrow 0} \int_{\Omega \backslash V_{\delta}} \mathrm{d} \mathbf{r}^{\prime} \mathrm{i} \omega \overline{\mathbf{G}}_{\mathbf{E}}\left(\mathbf{r}, \mathbf{r}^{\prime}\right) \cdot \Delta \epsilon\left(\mathbf{r}^{\prime}\right) \mathbf{E}\left(\mathbf{r}^{\prime}\right),
$$

where $\Omega$ is the volume of the scatterers, $\Delta \epsilon(\mathbf{r})$ describes the scatterers' dielectric constant difference from the background material, and $V_{\delta}$ is a small exclusion volume with size $\delta$ centered at $\mathbf{r}$. Therefore, one of the most difficult issues for VIE methods is how to compute accurately and efficiently the CPV for the dyadic Green's function with an $O\left(\frac{1}{R^{3}}\right)$ singularity, where $R$ is the distance between the source and field points.

As the CPV is defined through a limiting process of diminishing size $\delta$ of the exclusion volume $V_{\delta}$, in practical computation, a small finite $\delta>0$ has to be taken. Then, a simple and naive approximation could be written as

$$
\text { p.v. } \int_{\Omega} \mathrm{d} \mathbf{r}^{\prime} \mathrm{i} \omega \overline{\mathbf{G}}_{\mathbf{E}}\left(\mathbf{r}, \mathbf{r}^{\prime}\right) \cdot \Delta \epsilon\left(\mathbf{r}^{\prime}\right) \mathbf{E}\left(\mathbf{r}^{\prime}\right) \approx \int_{\Omega \backslash V_{\delta}} \mathrm{d} \mathbf{r}^{\prime} \mathrm{i} \omega \overline{\mathbf{G}}_{\mathbf{E}}\left(\mathbf{r}, \mathbf{r}^{\prime}\right) \cdot \Delta \epsilon\left(\mathbf{r}^{\prime}\right) \mathbf{E}\left(\mathbf{r}^{\prime}\right) .
$$

Here, we will be faced with two issues; it is the objective of this paper to address these two issues for the Nyström methods and find easily implementable solutions:

Firstly, we need to decide the size of $\delta$ to be taken and the effect of error by using a finite $\delta$ in the calculation of the CPV. As for any finite $\delta$, there will be a truncation error, which we name correction terms,

$$
\begin{aligned}
\text { p.v. } \int_{\Omega} \mathrm{d} \mathbf{r}^{\prime} \mathrm{i} \omega \overline{\mathbf{G}}_{\mathbf{E}}\left(\mathbf{r}, \mathbf{r}^{\prime}\right) \cdot \Delta \epsilon\left(\mathbf{r}^{\prime}\right) \mathbf{E}\left(\mathbf{r}^{\prime}\right) & =\int_{\Omega \backslash V_{\delta}} \mathrm{d} \mathbf{r}^{\prime} \mathrm{i} \omega \overline{\mathbf{G}}_{\mathbf{E}}\left(\mathbf{r}, \mathbf{r}^{\prime}\right) \cdot \Delta \epsilon\left(\mathbf{r}^{\prime}\right) \mathbf{E}\left(\mathbf{r}^{\prime}\right) \\
& + \text { correction terms. }
\end{aligned}
$$

Consequently, the correction terms will play an important role in determining the accurate numerical solution. The correction terms were derived by Fikioris [5] using a mixed potential formulation of the electric field. In this paper, we will re-derive the VIE using vector and scalar potentials such that the CPV can be computed with explicit expressions for the correction terms and the resulting VIE is in a form more suitable for numerical implementations. Previous work on how to handle singular integrals for VIE methods include singularity subtraction [8], locally corrected Nyström scheme [10], direct integration of the singularity [17], etc. 
Secondly, we need to find accurate and efficient ways to numerically compute the integral over $\Omega \backslash V_{\delta}$ for a fixed exclusion volume $V_{\delta}$.

To address the second issue, we will use special quadrature weights over tensor-product quadrature nodes in a reference element $\Omega$ (a sphere or a cube in this paper) by using an interpolation approach. Specifically, first a bruteforce computation of the integral, using Gauss quadrature in polar coordinate centered at the singularity, will be done to a given accuracy with a large number of evaluations of the integrand. The integrands in the VIE matrix entries, except for the singular denominators involving $R^{k}, 1 \leq k \leq 3$, are smooth functions, therefore they can be accurately interpolated using values only on a small number of the tensor-product nodes inside the domain $\Omega$. Then , the brute-force integration formula weights can be converted into new integration weights for the tensor-product nodes. Moreover, the new integration formula can be tabulated for integrating general functions. The Nyström collocation method based on the simple tensor-product nodes will be used to solve the VIE for the scattering of a large number of scatterers with a high accuracy using a minimal number of unknowns.

The rest of the paper is organized as follows: Section 2 presents the formulation of a VIE where the CPV can be computed with a finite exclusion volume $V_{\delta}$ accurately. Then, numerical algorithms are given in Section 3: the Nyström collocation methods, an efficient quadrature formula, and numerical implementation. Section 4 includes various numerical tests such as the accuracy of CPV computation, $\delta$-independence of matrix entries, $p$-refinement convergence for the VIE for spheres and cubes, and results of scattering from multiple scatterers. The paper ends with a conclusion in Section 5 .

\section{Volume Integral equations for Maxwell equations}

\subsection{VIE and Cauchy principal values}

In this section, we will follow [4] to show briefly how the VIE for the Maxwell equations can be derived using a vector form of Green's second identity for the following vector wave equations for the electric field $\mathbf{E}(\mathbf{r})$,

$$
\mathcal{L} \mathbf{E}(\mathbf{r})-\omega^{2} \epsilon(\mathbf{r}) \mathbf{E}(\mathbf{r})=-\mathrm{i} \omega \mathbf{J}_{e}(\mathbf{r}), \quad \mathbf{r} \in \mathbb{R}^{3} \backslash \partial \Omega
$$

where $\omega$ is the frequency, $\mu$ is the permeability, and $\mathcal{L}$ is a differential operator defined by

$$
\mathcal{L}=\nabla \times \frac{1}{\mu} \nabla \times
$$


source $\mathbf{J}_{e}(\mathbf{r})$ produces the incident wave impinging on the scatterers from above, i.e.,

$$
\mathbf{E}^{\mathrm{inc}}(\mathbf{r})=-\mathrm{i} \omega \mu(\mathbf{r}) \int_{\mathbb{R}^{3}} \overline{\mathbf{G}}_{E}\left(\mathbf{r}, \mathbf{r}^{\prime}\right) \cdot \mathbf{J}_{e}\left(\mathbf{r}^{\prime}\right) \mathrm{d} \mathbf{r}^{\prime},
$$

and $\overline{\mathbf{G}}_{E}\left(\mathbf{r}, \mathbf{r}^{\prime}\right)$ is the dyadic Green's function to be defined later. A scatterer $\Omega$ is characterized by a different dielectric constant from that of the background dielectrics $\epsilon_{L}(\mathbf{r})$, i.e.,

$$
\epsilon(\mathbf{r})=\epsilon_{L}(\mathbf{r})+\Delta \epsilon(\mathbf{r}),
$$

where $\Delta \epsilon(\mathbf{r})=0, \mathbf{r} \notin \Omega$. Then, (4) can be rewritten as

$$
\mathcal{L} \mathbf{E}(\mathbf{r})-\omega^{2} \epsilon_{L}(\mathbf{r}) \mathbf{E}(\mathbf{r})=-\mathrm{i} \omega \mathbf{J}(\mathbf{r}),
$$

where

$$
\mathbf{J}(\mathbf{r})=\mathbf{J}_{e}(\mathbf{r})+\mathbf{J}_{\text {eq }}(\mathbf{r}),
$$

and the equivalent current source $\mathbf{J}_{\text {eq }}(\mathbf{r})$ is defined to characterize the presence of the scatterer $\Omega$ :

$$
\mathbf{J}_{\text {eq }}(\mathbf{r})=\mathrm{i} \omega \Delta \epsilon(\mathbf{r}) \mathbf{E}(\mathbf{r})
$$

Let us consider any point $\mathbf{r}^{\prime} \in \Omega$ and a small volume $V_{\delta}=V_{\delta}\left(\mathbf{r}^{\prime}\right) \subset \Omega$ centered at $\mathbf{r}^{\prime}$. The dyadic Green's function $\overline{\mathbf{G}}_{E}\left(\mathbf{r}, \mathbf{r}^{\prime}\right)$ is defined by

$$
\mathcal{L} \overline{\mathbf{G}}_{E}\left(\mathbf{r}, \mathbf{r}^{\prime}\right)-\omega^{2} \epsilon_{L}(\mathbf{r}) \overline{\mathbf{G}}_{E}\left(\mathbf{r}, \mathbf{r}^{\prime}\right)=\frac{1}{\mu(\mathbf{r})} \overline{\mathbf{I}} \delta\left(\mathbf{r}-\mathbf{r}^{\prime}\right), \mathbf{r} \in \mathbb{R}^{3}
$$

In case of free-space, we have

$$
\overline{\mathbf{G}}_{\mathbf{E}}\left(\mathbf{r}, \mathbf{r}^{\prime}\right)=\overline{\mathbf{G}}_{\mathbf{E}}\left(\mathbf{r}^{\prime}, \mathbf{r}\right)=\left(\overline{\mathbf{I}}+\frac{1}{k^{2}} \nabla \nabla\right) g\left(\mathbf{r}, \mathbf{r}^{\prime}\right)
$$

where $k^{2}=\omega^{2} \epsilon_{L}(\mathbf{r}) \mu$ and

$$
g\left(\mathbf{r}, \mathbf{r}^{\prime}\right)=\frac{1}{4 \pi} \frac{\mathrm{e}^{-\mathrm{i} k R}}{R}, \quad R=\left|\mathbf{r}-\mathbf{r}^{\prime}\right|
$$

Next, we multiply (7) by $\overline{\mathbf{G}}_{E}\left(\mathbf{r}, \mathbf{r}^{\prime}\right)$ and 10 by $\mathbf{E}(\mathbf{r})$, form the difference, and integrate over the domain $\mathbb{R}^{3} \backslash V_{\delta}$. After some manipulation [4, we arrive at the following equation (after switching $\mathbf{r}$ and $\mathbf{r}^{\prime}$ ):

$$
\begin{array}{ll}
-\mathrm{i} \omega \mu(\mathbf{r}) \int_{\mathbb{R}^{3} \backslash V_{\delta}} \mathrm{d} \mathbf{r}^{\prime} \overline{\mathbf{G}}_{E}\left(\mathbf{r}, \mathbf{r}^{\prime}\right) \cdot \mathbf{J}\left(\mathbf{r}^{\prime}\right)-\mu(\mathbf{r}) \int_{S_{\delta}} \mathrm{d} s^{\prime}\left[\mathrm{i} \omega \overline{\mathbf{G}}_{E}\left(\mathbf{r}, \mathbf{r}^{\prime}\right) \cdot\left(\mathbf{n} \times \mathbf{H}\left(\mathbf{r}^{\prime}\right)\right)\right. \\
\left.-\frac{1}{\mu\left(\mathbf{r}^{\prime}\right)} \nabla \times \overline{\mathbf{G}}_{E}\left(\mathbf{r}, \mathbf{r}^{\prime}\right) \cdot\left(\mathbf{n} \times \mathbf{E}\left(\mathbf{r}^{\prime}\right)\right)\right]=\mathbf{0}, & \mathbf{r} \in \Omega,
\end{array}
$$

where $S_{\delta}=\partial V_{\delta}(\mathbf{r}), \mathbf{n}$ is the normal of $S_{\delta}$ pointing out of $V_{\delta}(\mathbf{r})$.

As $\delta \rightarrow 0$, the first integral will approach the CPV of a singular integral, while the surface integrals depends on the geometric shape of the volume $V_{\delta}$. 
In order to estimate the surface integrals, we have the following asymptotic approximations for $k R \ll 1[18]$ :

$$
\begin{aligned}
\overline{\mathbf{G}}_{\mathbf{E}}\left(\mathbf{r}, \mathbf{r}^{\prime}\right) & =\frac{1}{4 \pi k^{2} R^{3}}(\mathbf{I}-3 \mathbf{u} \otimes \mathbf{u})+O\left(\frac{1}{R^{2}}\right), \\
\nabla^{\prime} \times \overline{\mathbf{G}}_{\mathbf{E}}\left(\mathbf{r}, \mathbf{r}^{\prime}\right) & =\frac{1}{4 \pi R^{2}} \mathbf{u} \times \mathbf{I}+O\left(\frac{1}{R}\right),
\end{aligned}
$$

where $\mathbf{u}=\left(\mathbf{r}^{\prime}-\mathbf{r}\right) / R$, which implies that:

$$
\begin{aligned}
& \lim _{\delta \rightarrow 0} \int_{S_{\delta}} \mathrm{d} s^{\prime} \mathbf{n} \times \mathbf{E}\left(\mathbf{r}^{\prime}\right) \cdot \nabla \times \overline{\mathbf{G}}_{E}\left(\mathbf{r}^{\prime}, \mathbf{r}\right)=-\left[\mathbf{I}-\mathbf{L}_{V_{\delta}}\right] \cdot \mathbf{E}(\mathbf{r}), \\
& \lim _{\delta \rightarrow 0} \int_{S_{\delta}} \mathrm{d} s^{\prime} \mathbf{n} \times \mathbf{H}\left(\mathbf{r}^{\prime}\right) \cdot \overline{\mathbf{G}}_{E}\left(\mathbf{r}^{\prime}, \mathbf{r}\right)=-\frac{1}{k^{2}} \mathbf{L}_{V_{\delta}} \cdot \nabla \times \mathbf{H}(\mathbf{r}),
\end{aligned}
$$

and the $\mathbf{L}$-dyadics for $V_{\delta}$ of various geometric shapes are given in [20]. We have

$$
\mathbf{L}_{V_{\delta}}=\frac{1}{3} \mathbf{I}
$$

for a sphere as used in this paper.

Substituting (16) and (17) into (13), and using Ampère's law, we have the VIE for the electric field for $\mathbf{r} \in \Omega$ :

$$
\mathbf{C} \cdot \mathbf{E}(\mathbf{r})=\mathbf{E}^{\mathrm{inc}}(\mathbf{r})-\mathrm{i} \omega \mu(\mathbf{r}) \text { p.v. } \int_{\Omega} \mathrm{d} \mathbf{r}^{\prime} \mathrm{i} \omega \overline{\mathbf{G}}_{\mathbf{E}}\left(\mathbf{r}, \mathbf{r}^{\prime}\right) \cdot \Delta \epsilon\left(\mathbf{r}^{\prime}\right) \mathbf{E}\left(\mathbf{r}^{\prime}\right),
$$

where the coefficient matrix is given by

$$
\mathbf{C}=\mathbf{I}+\mathbf{L}_{V_{\delta}} \cdot \Delta \epsilon(\mathbf{r}) .
$$

As mentioned in the introduction, in most numerical implementations of (19), the CPV integral is computed by selecting a finite $\delta$ as in (2). The numerical solution thus obtained does not satisfy the original VIE (19) and will have an intrinsic error related to the correction terms in (3).

\subsection{Reformulation of the VIE and computing CPV with a finite $\delta$}

In this section, we will re-derive the VIE for the electric field where the CPV in (20) can be computed with a finite exclusion volume $V_{\delta}$ together with

some correction terms. Based on the Helmholtz decomposition, the electric field $\mathbf{E}(\mathbf{r})$ can be expressed as follows [4,

$$
\mathbf{E}=-\mathrm{i} \omega \mathbf{A}-\nabla V
$$


where $A$ and $V$ are vector and scalar potentials, respectively. Using the Lorentz gauge condition [16],

$$
\nabla \cdot \mathbf{A}=-\mathrm{i} \omega \epsilon \mu V
$$

we can have a vector potential representation

$$
\mathbf{E}=-\mathrm{i} \omega \mathbf{A}+\frac{1}{i \omega \epsilon \mu} \nabla(\nabla \cdot \mathbf{A})=-i \omega\left[\overline{\mathbf{I}}+\frac{1}{k^{2}} \nabla \nabla\right] \mathbf{A}
$$

Meanwhile, it can be shown that the potential A satisfies the Helmholtz equation component-wise [16],

$$
\nabla^{2} \mathbf{A}+k^{2} \mathbf{A}=-\mu \mathbf{J}
$$

Thus, the solution A of Eq. 23) can be rewritten in an integral representation:

$$
\begin{aligned}
\mathbf{A} & =\mu \int_{\mathbb{R}^{3}} \mathrm{~d} \mathbf{r}^{\prime} \mathbf{J}\left(\mathbf{r}^{\prime}\right) g\left(\mathbf{r}, \mathbf{r}^{\prime}\right) \\
& =\mu \int_{\mathbb{R}^{3} \backslash \Omega} \mathbf{J}_{e}\left(\mathbf{r}^{\prime}\right) g\left(\mathbf{r}, \mathbf{r}^{\prime}\right) \mathrm{d} \mathbf{r}^{\prime}+\mu \int_{\Omega} \mathbf{J}_{\mathrm{eq}}\left(\mathbf{r}^{\prime}\right) g\left(\mathbf{r}, \mathbf{r}^{\prime}\right) \mathrm{d} \mathbf{r}^{\prime} \\
& =\mu \int_{\mathbb{R}^{3} \backslash \Omega} \mathbf{J}_{e}\left(\mathbf{r}^{\prime}\right) g\left(\mathbf{r}, \mathbf{r}^{\prime}\right) \mathrm{d} \mathbf{r}^{\prime}+\mu \int_{\Omega} \mathrm{d} \mathbf{r}^{\prime} \mathrm{i} \omega \Delta \epsilon\left(\mathbf{r}^{\prime}\right) \mathbf{E}\left(\mathbf{r}^{\prime}\right) g\left(\mathbf{r}, \mathbf{r}^{\prime}\right),
\end{aligned}
$$

where the second equality on the right hand side of Eq. (24) is due to the assumption that $\operatorname{supp}\left(\mathbf{J}_{e}(\mathbf{r})\right) \cap \Omega=\emptyset$.

The first integral of (24) is well-defined if $\mathbf{r} \in \Omega$ and after being plugged it into Eq. (22), it yields the incident wave $\mathbf{E}^{\text {inc }}(\mathbf{r})$ according to relation (5). For the second integral over $\Omega$, we split it as follows:

$$
\mu \int_{\Omega} \mathrm{d} \mathbf{r}^{\prime} \mathrm{i} \omega \Delta \epsilon\left(\mathbf{r}^{\prime}\right) \mathbf{E}\left(\mathbf{r}^{\prime}\right) g\left(\mathbf{r}, \mathbf{r}^{\prime}\right)=\mu\left(\int_{\Omega \backslash V_{\delta}}+\int_{V_{\delta}}\right) \mathrm{i} \omega \Delta \epsilon\left(\mathbf{r}^{\prime}\right) \mathbf{E}\left(\mathbf{r}^{\prime}\right) g\left(\mathbf{r}, \mathbf{r}^{\prime}\right),
$$

and along with the first integral term in Eq. (24), it follows from Eq. (22) that

$$
\begin{aligned}
\mathbf{E} & =\mathbf{E}^{\mathrm{inc}}(\mathbf{r})-\mathrm{i} \omega \mu \int_{\Omega \backslash V_{\delta}} \mathrm{i} \omega \Delta \epsilon\left(\mathbf{r}^{\prime}\right) \mathbf{E}\left(\mathbf{r}^{\prime}\right)\left[\overline{\mathbf{I}}+\frac{1}{k^{2}} \nabla \nabla\right] g\left(\mathbf{r}, \mathbf{r}^{\prime}\right) \\
& -\mathrm{i} \omega \mu\left[\overline{\mathbf{I}}+\frac{1}{k^{2}} \nabla \nabla\right] \int_{V_{\delta}} \mathrm{d} \mathbf{r}^{\prime} \mathrm{i} \omega \Delta \epsilon\left(\mathbf{r}^{\prime}\right) \mathbf{E}\left(\mathbf{r}^{\prime}\right) g\left(\mathbf{r}, \mathbf{r}^{\prime}\right) \\
& =\mathbf{E}^{\mathrm{inc}}(\mathbf{r})-\mathrm{i} \omega \mu \int_{\Omega \backslash V_{\delta}} \mathrm{i} \omega \Delta \epsilon\left(\mathbf{r}^{\prime}\right) \overline{\mathbf{G}}_{\mathbf{E}}\left(\mathbf{r}, \mathbf{r}^{\prime}\right) \cdot \mathbf{E}\left(\mathbf{r}^{\prime}\right) \\
& -\mathrm{i} \omega \mu\left[\overline{\mathbf{I}}+\frac{1}{k^{2}} \nabla \nabla\right] \int_{V_{\delta}} \mathrm{d} \mathbf{r}^{\prime} \mathrm{i} \omega \Delta \epsilon\left(\mathbf{r}^{\prime}\right) \mathbf{E}\left(\mathbf{r}^{\prime}\right) g\left(\mathbf{r}, \mathbf{r}^{\prime}\right) .
\end{aligned}
$$

Next, we separate the singular part in $g\left(\mathbf{r}, \mathbf{r}^{\prime}\right)$ as follows:

$$
g\left(\mathbf{r}, \mathbf{r}^{\prime}\right)=g_{0}\left(\mathbf{r}, \mathbf{r}^{\prime}\right)+\widetilde{g}\left(\mathbf{r}, \mathbf{r}^{\prime}\right)
$$


where

$$
g_{0}\left(\mathbf{r}, \mathbf{r}^{\prime}\right)=\frac{1}{4 \pi\left|\mathbf{r}-\mathbf{r}^{\prime}\right|}, \quad \widetilde{g}=g-g_{0} .
$$

Then, using the fact that [9,20]

$$
\nabla \nabla \int_{V_{\delta}} \mathrm{d} \mathbf{r}^{\prime} \frac{1}{4 \pi\left|\mathbf{r}-\mathbf{r}^{\prime}\right|}=-\int_{\partial V_{\delta}} \mathrm{d} s^{\prime} \frac{\left(\mathbf{r}-\mathbf{r}^{\prime}\right) \mathbf{u}_{n}\left(\mathbf{r}^{\prime}\right)}{4 \pi\left|\mathbf{r}-\mathbf{r}^{\prime}\right|^{3}}=-\mathbf{L}_{V_{\delta}},
$$

we can compute the following integral as

$$
\begin{aligned}
& \nabla \nabla \int_{V_{\delta}} \mathrm{d} \mathbf{r}^{\prime} \Delta \epsilon\left(\mathbf{r}^{\prime}\right) \mathbf{E}\left(\mathbf{r}^{\prime}\right) g_{0}\left(\mathbf{r}, \mathbf{r}^{\prime}\right) \\
& =\nabla \nabla \int_{V_{\delta}} \mathrm{d} \mathbf{r}^{\prime} \frac{1}{4 \pi\left|\mathbf{r}-\mathbf{r}^{\prime}\right|} \Delta \epsilon(\mathbf{r}) \mathbf{E}(\mathbf{r})+\int_{V_{\delta}} \mathrm{d} \mathbf{r}^{\prime} \nabla \nabla g_{0}\left(\mathbf{r}, \mathbf{r}^{\prime}\right)\left[\Delta \epsilon\left(\mathbf{r}^{\prime}\right) \mathbf{E}\left(\mathbf{r}^{\prime}\right)-\Delta \epsilon(\mathbf{r}) \mathbf{E}(\mathbf{r})\right] \\
& =-\mathbf{L}_{V_{\delta}} \Delta \epsilon(\mathbf{r}) \mathbf{E}(\mathbf{r})+\int_{V_{\delta}} \mathrm{d} \mathbf{r}^{\prime} \nabla \nabla g_{0}\left(\mathbf{r}, \mathbf{r}^{\prime}\right)\left[\Delta \epsilon\left(\mathbf{r}^{\prime}\right) \mathbf{E}\left(\mathbf{r}^{\prime}\right)-\Delta \epsilon(\mathbf{r}) \mathbf{E}(\mathbf{r})\right]
\end{aligned}
$$

where the second integral has a removable singularity $O\left(\frac{1}{\left|\mathbf{r}-\mathbf{r}^{\prime}\right|^{2}}\right)$ through the use of spherical coordinates centered at $\mathbf{r}$, provided the function $\Delta \epsilon(\mathbf{r}) \mathbf{E}(\mathbf{r})$ is differentiable in the interior of $V_{\delta}$, which we assume it to be.

With 29) and the fact that $\tilde{g}=g-g_{0}$ is a smooth function, 25 becomes

$$
\begin{aligned}
\mathbf{C} \cdot \mathbf{E} & =\mathbf{E}^{\mathrm{inc}}(\mathbf{r})-i \omega \mu \int_{\Omega \backslash V_{\delta}} i \omega \Delta \epsilon\left(\mathbf{r}^{\prime}\right) \overline{\mathbf{G}_{\mathbf{E}}}\left(\mathbf{r}, \mathbf{r}^{\prime}\right) \cdot \mathbf{E}\left(\mathbf{r}^{\prime}\right) \\
& +\omega^{2} \mu \int_{V_{\delta}} \mathrm{d} \mathbf{r}^{\prime} \Delta \epsilon\left(\mathbf{r}^{\prime}\right) \mathbf{E}\left(\mathbf{r}^{\prime}\right) g\left(\mathbf{r}, \mathbf{r}^{\prime}\right) \\
& +\frac{\omega^{2}}{k^{2}} \mu \int_{V_{\delta}} \mathrm{d} \mathbf{r}^{\prime} \Delta \epsilon\left(\mathbf{r}^{\prime}\right) \nabla \nabla \widetilde{g}\left(\mathbf{r}, \mathbf{r}^{\prime}\right) \cdot \mathbf{E}\left(\mathbf{r}^{\prime}\right) \\
& +\frac{\omega^{2}}{k^{2}} \mu \int_{V_{\delta}} \mathrm{d} \mathbf{r}^{\prime} \nabla \nabla g_{0}\left(\mathbf{r}, \mathbf{r}^{\prime}\right)\left[\Delta \epsilon\left(\mathbf{r}^{\prime}\right) \mathbf{E}\left(\mathbf{r}^{\prime}\right)-\Delta \epsilon(\mathbf{r}) \mathbf{E}(\mathbf{r})\right]
\end{aligned}
$$

with same coefficient $\mathbf{C}$ as in 20 .

The VIE in (30) is similar to those obtained by Fikioris [5]; however, our derivation is based on a splitting of Green's function in (26) and the identity for $\mathbf{L}_{V_{\delta}}$ in (28). A comparison study between CPV formulation (19) and finite exclusion volume formulation (30) can be found in [19. Now expression (30) holds for any finite $\delta>0$ as long as $V_{\delta} \subset \Omega$, and all integrals involved on the right-hand side are well-defined provided that $\Delta \epsilon(\mathbf{r}) \mathbf{E}(\mathbf{r})$ is Hölder continuous. We can see that the last three integrals can be understood as the correction terms for computing the Cauchy principal value with a finite-sized exclusion volume $V_{\delta}$. It should be noted that these integrals are all weakly singular integrals whose singularities can be removed by a spherical coordinate transform. In particular, we can estimate their magnitudes in terms of $\delta$. Namely,

$$
\left|\int_{V_{\delta}} \mathrm{d} \mathbf{r}^{\prime} \Delta \epsilon\left(\mathbf{r}^{\prime}\right) \mathbf{E}\left(\mathbf{r}^{\prime}\right) g\left(\mathbf{r}, \mathbf{r}^{\prime}\right)\right| \leq C_{1}\|\Delta \epsilon \mathbf{E}\|_{\infty} \delta^{2}
$$




$$
\left|\int_{V_{\delta}} \mathrm{d} \mathbf{r}^{\prime} \Delta \epsilon\left(\mathbf{r}^{\prime}\right) \nabla \nabla \widetilde{g}\left(\mathbf{r}, \mathbf{r}^{\prime}\right) \cdot \mathbf{E}\left(\mathbf{r}^{\prime}\right)\right| \leq C_{2}\|\Delta \epsilon \mathbf{E}\|_{\infty} \delta^{2}
$$

and

$$
\left|\int_{V_{\delta}} \mathrm{d} \mathbf{r}^{\prime} \nabla \nabla g_{0}\left(\mathbf{r}, \mathbf{r}^{\prime}\right)\left[\Delta \epsilon\left(\mathbf{r}^{\prime}\right) \mathbf{E}\left(\mathbf{r}^{\prime}\right)-\Delta \epsilon(\mathbf{r}) \mathbf{E}(\mathbf{r})\right]\right| \leq C_{3}\|\Delta \epsilon \mathbf{E}\|_{1, \infty} \delta
$$

where $C_{1}, C_{2}, C_{3}$ are constants, and $\|\cdot\|_{\infty}$ and $\|\cdot\|_{1, \infty}$ represent the $L^{\infty}$ norms of a function and its first derivative, respectively.

Remark 1 Equations (31)-(33) explicitly show the accuracy of approximating the $C P V$ (1) by the integral (2) with a finite $\delta>0$, i.e., the truncation error is of the order $O(\delta)$. Hence the numerical solution of the VIE will have this $O(\delta)$ truncation error in general regardless of the integration quadratures used if terms in (31)-(33) are not included.

Moreover, in case that $V_{\delta}$ is a ball of radius $\delta$ centered at $\mathbf{r}$, one can obtain a better estimate than (33) due to the anti-symmetry of the singular term $\nabla \nabla g_{0}\left(\mathbf{r}, \mathbf{r}^{\prime}\right)$ in the spherical coordinates, i.e.

$$
\left|\int_{V_{\delta}} \mathrm{d} \mathbf{r}^{\prime} \nabla \nabla g_{0}\left(\mathbf{r}, \mathbf{r}^{\prime}\right)\left[\Delta \epsilon\left(\mathbf{r}^{\prime}\right) \mathbf{E}\left(\mathbf{r}^{\prime}\right)-\Delta \epsilon(\mathbf{r}) \mathbf{E}(\mathbf{r})\right]\right| \leq C_{4}\|\Delta \epsilon \mathbf{E}\|_{2, \infty} \delta^{2}
$$

where $C_{4}$ are constant.

\section{Numerical Methods}

\subsection{Nyström collocation method}

We use Nyström collocation methods to solve (30). First, we assume the computational domain $\Omega$ comprised of an $N$-number non-overlapping elements (cubes and balls) $\Omega_{i}, i=1,2, \ldots, N$. On each element $\Omega_{i}$, we as$\operatorname{sign} M$ tensor-product nodes for which $M$ scalar interpolant basis functions $\phi_{i j}, j=1,2,3, \ldots . M$ are defined. Then, we can write the solution as

$$
\mathbf{E}(\mathbf{r})=\sum_{i=1}^{N} \sum_{j=1}^{M} \mathbf{c}_{i j} \phi_{i j}(\mathbf{r}), \quad \mathbf{r} \in \Omega_{i}
$$


where $\mathbf{c}_{i j}$ are the unknown vectorial coefficients. Inserting (35) into (30), we obtain the following equations for $\mathbf{c}_{i j}$ :

$$
\begin{aligned}
\mathbf{C} \cdot \mathbf{c}_{i j} & =\mathbf{E}_{i j}^{\mathrm{inc}}+\omega^{2} \mu \sum_{n=1}^{N} \sum_{m=1}^{M}\left[\int_{\Omega_{n} \backslash V_{\delta_{i j}}} \mathrm{~d} \mathbf{r}^{\prime} \Delta \epsilon\left(\mathbf{r}^{\prime}\right) \overline{\mathbf{G}}_{\mathbf{E}}\left(\mathbf{r}_{i j}, \mathbf{r}^{\prime}\right) \phi_{n m}\left(\mathbf{r}^{\prime}\right)\right] \cdot \mathbf{c}_{n m} \\
& +\omega^{2} \mu \sum_{m=1}^{M}\left[\int_{V_{\delta_{i j}}} \mathrm{~d} \mathbf{r}^{\prime} \Delta \epsilon\left(\mathbf{r}^{\prime}\right) g\left(\mathbf{r}_{i j}, \mathbf{r}^{\prime}\right) \phi_{i m}\left(\mathbf{r}^{\prime}\right)\right] \cdot \mathbf{c}_{i m} \\
& +\frac{\omega^{2} \mu}{k^{2}} \sum_{m=1}^{M}\left[\int_{V_{\delta_{i j}}} \mathrm{~d} \mathbf{r}^{\prime} \Delta \epsilon\left(\mathbf{r}^{\prime}\right) \nabla \nabla \widetilde{g}\left(\mathbf{r}_{i j}, \mathbf{r}^{\prime}\right) \phi_{i m}\left(\mathbf{r}^{\prime}\right)\right] \cdot \mathbf{c}_{i m} \\
& +\frac{\omega^{2} \mu}{k^{2}} \sum_{m=1}^{M} \int_{V_{\delta_{i j}}} \mathrm{~d} \mathbf{r}^{\prime} \nabla^{2} g_{0}\left(\mathbf{r}_{i j}, \mathbf{r}^{\prime}\right)\left[\Delta \epsilon\left(\mathbf{r}^{\prime}\right) \phi_{i m}\left(\mathbf{r}^{\prime}\right)-\Delta \epsilon_{i j} \phi_{i m}\left(\mathbf{r}_{i j}\right)\right] \cdot \mathbf{c}_{i m} .
\end{aligned}
$$

When $n \neq i$, we have $V_{\delta_{i j}} \notin \Omega$, then the first integral of (36)

$$
\int_{\Omega_{n} \backslash V_{\delta_{i j}}} \mathrm{~d} \mathbf{r}^{\prime} \Delta \epsilon\left(\mathbf{r}^{\prime}\right) \overline{\mathbf{G}}_{\mathbf{E}}\left(\mathbf{r}_{i j}, \mathbf{r}^{\prime}\right) \phi_{n m}\left(\mathbf{r}^{\prime}\right)
$$

is regular in the whole domain $\Omega_{n}$ and hence it can be evaluated by regular Gauss quadratures, i.e.,

$$
\int_{\Omega_{n}} \mathrm{~d} \mathbf{r}^{\prime} \Delta \epsilon\left(\mathbf{r}^{\prime}\right) \overline{\mathbf{G}}_{\mathbf{E}}\left(\mathbf{r}_{i j}, \mathbf{r}^{\prime}\right) \phi_{n m}\left(\mathbf{r}^{\prime}\right)=\left(\frac{a_{n}}{2}\right)^{3} \sum_{m=1}^{M} \Delta \epsilon_{n m} \overline{\mathbf{G}}_{\mathbf{E}}\left(\mathbf{r}_{i j}, \mathbf{r}_{n m}\right) \omega_{m}^{s},
$$

with $\omega_{m}^{s}$ being the standard Gauss weights in 3-D, which are obtained from the tensor product of the Gauss weights in $[-1,1]$ or uniform weights for the periodic direction in $\phi \in[0,2 \pi]$ in the cases of spheres.

When $n=i$, although the singularity $\mathbf{r}_{i j}$ is excluded from the domain $\Omega_{i}$, the calculation of the integral

$$
\int_{\Omega_{i} \backslash V_{\delta_{i j}}} \mathrm{~d} \mathbf{r}^{\prime} \Delta \epsilon\left(\mathbf{r}^{\prime}\right) \overline{\mathbf{G}}_{\mathbf{E}}\left(\mathbf{r}_{i j}, \mathbf{r}^{\prime}\right) \phi_{i m}\left(\mathbf{r}^{\prime}\right)
$$

is still challenging. Therefore, we present an efficient quadrature formula to evaluate this integral in the following subsection.

\subsection{Interpolated weights on tensor-product nodes for integrals on $\Omega \backslash V_{\delta}$}

For the sake of generality, we consider the following integral:

$$
I_{s}=\int_{\Omega \backslash V_{\delta}} \frac{f\left(\mathbf{r} ; \mathbf{r}^{\prime}\right) h\left(\mathbf{r} ; \mathbf{r}^{\prime}\right)}{R^{k}} \mathrm{~d} \mathbf{r}^{\prime}, \quad \mathbf{r} \in V_{\delta},
$$


where $k=1,2,3$ corresponds to weak-, strong-, and hyper-singularity of the integral, respectively. The function $f\left(\mathbf{r} ; \mathbf{r}^{\prime}\right)$ is assumed to be a general smooth and well-defined function, while $h\left(\mathbf{r} ; \mathbf{r}^{\prime}\right)$ is some fixed function resulting from the directional derivative in the definition of the dyadic Green's function.

Because the function $f\left(\mathbf{r} ; \mathbf{r}^{\prime}\right)$ is smooth over the whole domain $\Omega$, it can be well approximated by the following simple interpolation:

$$
f\left(\mathbf{r} ; \mathbf{r}^{\prime}\right) \approx \sum_{j=1}^{J} f\left(\mathbf{r} ; \mathbf{r}_{j}\right) \phi_{j}\left(\mathbf{r}^{\prime}\right), \quad \mathbf{r}_{j} \in \Omega
$$

where $\left\{\mathbf{r}_{j}\right\}_{j=1}^{J}$ are $J$-nodes in $\Omega$ formed by a tensor product of Gauss nodes in $[-1,1]$ or uniformly spaced nodes in $[0,2 \pi]$ for periodic direction. Replacing $f\left(\mathbf{r} ; \mathbf{r}^{\prime}\right)$ in 40 with 41 yields

$$
\int_{\Omega \backslash V_{\delta}} \frac{f\left(\mathbf{r} ; \mathbf{r}^{\prime}\right) h\left(\mathbf{r} ; \mathbf{r}^{\prime}\right)}{R^{k}} d \mathbf{r}^{\prime} \approx \sum_{j=1}^{J} f\left(\mathbf{r} ; \mathbf{r}_{j}\right) \omega_{j},
$$

We call $\omega_{j}$ the interpolated weights, or just interpolated weights, defined through the integral

$$
\omega_{j}=\int_{\Omega \backslash V_{\delta}} \frac{\phi_{j}\left(\mathbf{r}^{\prime}\right) h\left(\mathbf{r} ; \mathbf{r}^{\prime}\right)}{\left|\mathbf{r}-\mathbf{r}^{\prime}\right|^{k}} d \mathbf{r}^{\prime} .
$$

Note that the interpolated weights $\omega_{j}$ depend on the location of the singularity $\mathbf{r}$ and relies on accurate calculations of (43), which can be accomplished in two steps: for the case of a cube domain $\Omega$, first $\Omega$ is subdivided upto 18 subcubes (the number of subcubes depends on the location of the singularity) with one containing the singularity $\mathbf{r}$ in its center. For the cube including the singularity, a straight-forward, brute-force approach involving a large number $N_{b}\left(N_{b} \gg J\right)$ of Gauss points is adopted in local spherical polar coordinates to obtain satisfactory accuracy. While for the other cubes, regular tensor product Gauss quadrature is applied. Details of the computations and resulting weight $\left\{\omega_{j}\right\}$ tables and also similar approaches for sphere and cylinder domains could be found in [21].

The computation of weights $\omega_{j}$ only needs to be performed once and tabulated for the reference domain; they can be used for general cubic or spherical domains. Due to the smoothness of function $f\left(\mathbf{r}, \mathbf{r}^{\prime}\right)$, the number $J$ is relatively small, especially if the element size is small as in meta-material designs, so the computation of (39) is efficient once $\omega_{j}$ are obtained.

Remark 2 As the $C P V$ is used in the VIE, the VIE depends on the specific shape of the exclusion volume. Thus, the interpolated weights can only be used for the shape for which it was calculated, namely, a cube or a sphere shape here. So, for a general element obtained by an affine mapping as in a finite element triangulation, we will need to isolate the singularity by a cube or a 
sphere with the singularity at its center, then the pre-calculated interpolated weights defined in (43) can be used where integral over the rest of the region within the element of a more general shape can be computed with regular Gauss quadratures.

\subsection{Computation of VIE matrix entries}

In this section, we will show how to compute the matrix entries accurately for the VIE in the following steps.

- Step I: calculate interpolated weights $\left\{\omega_{j}\right\}$ on the reference (cubic or spherical) domain.

For Eq. (43), we take $V_{\delta}=B\left(\mathbf{r}_{j}, \delta\right)$, where $\delta>0$ is a prescribed small quantity and $\mathbf{r}_{j}, j=1,2, \ldots M$ are the $M$ tensor-product nodes in the reference domain, and we let $J=M$ in Eq. (41).

Next, we have the dyadic Green's function for the free-space in the following form,

$$
\begin{aligned}
\overline{\mathbf{G}}_{\mathbf{E}} & =g \mathbf{I}+\frac{\nabla^{2} g}{k^{2}}=\frac{e^{-i k R}}{4 \pi R}(\mathbf{I}-\mathbf{u} \otimes \mathbf{u}) \\
& -\frac{i e^{-i k R}}{4 \pi R^{2} k}(\mathbf{I}-3 \mathbf{u} \otimes \mathbf{u})-\frac{e^{-i k R}}{4 \pi R^{3} k^{2}}(\mathbf{I}-3 \mathbf{u} \otimes \mathbf{u}) .
\end{aligned}
$$

Since the value of function $\mathbf{u} \otimes \mathbf{u}$ is multi-valued at $R=0$, in 40 we will take

$$
h\left(\mathbf{r} ; \mathbf{r}^{\prime}\right)=\mathbf{u} \otimes \mathbf{u},
$$

and hence 43) will produce a set of 9 interpolated weights. However, due to the symmetry of the matrix $\mathbf{u} \otimes \mathbf{u}$, only 6 components need to be considered. For the identity matrix I term in (44), we need a set of scalar interpolation weights by assuming $h\left(\mathbf{r}, \mathbf{r}^{\prime}\right)=1$ in (43).

Additionally, for the scalar and matrix weights, we will consider $k=1,2$, and 3 for weak, strong, and hyper singular integrals, respectively.

In summary, for each collocation point (also the singularity location) $\mathbf{r}_{j}, j=1,2, \ldots, M$ in an element, scalar weights $\omega_{j, m}, \bar{\omega}_{j, m}$ and $\tilde{\omega}_{j, m}, m=$ $1,2, \ldots, M$ are calculated for weak-, strong-, hyper-singular integrals, respectively. And the corresponding matrix weights are denoted as $\Lambda_{j, m}, \bar{\Lambda}_{j, m}$, and $\tilde{\Lambda}_{j, m}$. Here the first index $j$ indicates the location of the singularity of the integrand while the second one $m$ is the quadrature weight index. These weights only need to be calculated once and then stored for future use.

- Step II: We consider the computational domain consisting of a collection of fundamental elements with size $a_{i}, i=1,2, \ldots, N$ and assign $M$ collocation 
points in each elements. For the $j$-th collocation point $\mathbf{r}_{i j}$ in the $i$-th element, we construct the equation:

$$
-\omega^{2} \mu \sum_{n=1}^{N} \sum_{m=1}^{M} \mathbf{A}_{n m} \cdot \mathbf{c}_{n m}-\sum_{m=1}^{M} \mathbf{B}_{i m} \cdot \mathbf{c}_{i m}+\left(1+\frac{1}{3} \Delta \epsilon_{i j}\right) \mathbf{I}_{3 \times 3} \cdot \mathbf{c}_{i j}=\mathbf{E}_{i j}^{\mathrm{inc}}
$$

The matrix $\mathbf{B}$ originates from the correction terms of the Cauchy principal value

$$
\begin{aligned}
\mathbf{B}_{i m} & =\omega^{2} \mu \int_{B\left(\mathbf{r}_{i j}, a_{i} \delta\right)} \mathrm{d} \mathbf{r}^{\prime} \Delta \epsilon\left(\mathbf{r}^{\prime}\right) g\left(\mathbf{r}_{i j}, \mathbf{r}^{\prime}\right) \phi_{i m}\left(\mathbf{r}^{\prime}\right) \\
& +\frac{\omega^{2} \mu}{k^{2}} \int_{B\left(\mathbf{r}_{i j}, a_{i} \delta\right)} \mathrm{d} \mathbf{r}^{\prime} \Delta \epsilon\left(\mathbf{r}^{\prime}\right) \nabla^{2} \tilde{g}\left(\mathbf{r}_{i j}, \mathbf{r}^{\prime}\right) \phi_{i m}\left(\mathbf{r}^{\prime}\right) \\
& \left.+\frac{\omega^{2} \mu}{k^{2}} \int_{B\left(\mathbf{r}_{i j}, a_{i} \delta\right)} \mathrm{d} \mathbf{r}^{\prime} \nabla^{2} g_{0}\left(\mathbf{r}_{i j}, \mathbf{r}^{\prime}\right)\left[\Delta \epsilon\left(\mathbf{r}^{\prime}\right) \phi_{i m}\left(\mathbf{r}^{\prime}\right)-\Delta \epsilon_{i j} \phi_{i m}\left(\mathbf{r}_{i j}\right)\right)\right]
\end{aligned}
$$

and it can be calculated by standard Gauss quadrature through spherical coordinates since the Jacobian will eliminate completely the singularity of the integrands.

When $n=i$, we calculate the integral (39) as

$$
\begin{aligned}
\mathbf{A}_{i m} & =\frac{1}{4 \pi} J_{i} \sum_{j=1}^{M} \Delta \epsilon_{i m}\left[\left(e^{-i k R_{m}} \omega_{j, m}^{i}-i e^{-i k R_{m}} \bar{\omega}_{j, m}^{i}-e^{-i k R_{m}} \tilde{\omega}_{j, m}^{i}\right) \mathbf{I}_{3 \times 3}\right. \\
& \left.e^{-i k R_{m}} \Lambda_{j, m}^{i}-i e^{-i k R_{m}} \bar{\Lambda}_{j, m}^{i}-e^{-i k R_{m}} \tilde{\Lambda}_{j, m}^{i}\right]
\end{aligned}
$$

where $R_{m}=\left|\mathbf{r}_{i j}-\mathbf{r}_{i m}\right|$ and $J_{i}$ is the Jacobian from the reference domain to the physical domain $\Omega_{i}$.

Note that the interpolated quadrature weights are rescaled from the reference domain. In the cube example, the reference domain is $[-1,1]^{3}$ and if the physical domain has length $a_{i}$, then $J_{i}=\frac{a_{i}}{2}$ and recall the definition of the interpolated weights (43), we have

$$
\begin{aligned}
& \omega_{j, m}^{i}=\left(\frac{2}{a_{i}}\right) \omega_{j, m}, \bar{\omega}_{j, m}^{i}=\left(\frac{2}{a_{i}}\right)^{2} \bar{\omega}_{j, m}, \tilde{\omega}_{j, m}^{i}=\left(\frac{2}{a_{i}}\right)^{3} \tilde{\omega}_{j, m} \\
& \Lambda_{m}^{i}=\left(\frac{2}{a_{i}}\right) \Lambda_{j, m}, \quad \bar{\Lambda}_{j, m}^{i}=\left(\frac{2}{a_{i}}\right)^{2} \bar{\Lambda}_{j, m}, \tilde{\Lambda}_{j, m}^{i}=\left(\frac{2}{a_{i}}\right)^{3} \tilde{\Lambda}_{j, m}
\end{aligned}
$$

when $n \neq i$, we have

$$
\mathbf{A}_{n m}=J_{n} \sum_{j=1}^{M} \Delta \epsilon_{n m} \overline{\mathbf{G}}_{\mathbf{E}}\left(\mathbf{r}_{i j}, \mathbf{r}_{n m}\right) \omega_{j}^{s}
$$

- Step III: (46) for all the $N \times M$ tensor-product nodes can be assembled as 
the following linear algebraic equation system

$$
\mathbf{V} \cdot \overrightarrow{\mathbf{c}}=\left[\begin{array}{ccc}
\mathbf{V}_{x x} & \mathbf{V}_{x y} & \mathbf{V}_{x z} \\
\mathbf{V}_{y x} & \mathbf{V}_{y y} & \mathbf{V}_{y z} \\
\mathbf{V}_{z x} & \mathbf{V}_{z y} & \mathbf{V}_{z z}
\end{array}\right] \cdot\left[\begin{array}{c}
\mathbf{c}_{x} \\
\mathbf{c}_{y} \\
\mathbf{c}_{z}
\end{array}\right]=\left[\begin{array}{c}
\mathbf{E}_{x}^{\mathrm{inc}} \\
\mathbf{E}_{y}^{\mathrm{inc}} \\
\mathbf{E}_{z}^{\mathrm{inc}}
\end{array}\right] .
$$

Based on the properties of the Green's function, the $3 N M \times 3 N M$ matrix $\mathrm{V}$ is partitioned into nine blocks, each block is a $N M \times N M$ sub-matrix. The solution of the VIE contains three $N M \times 1$ vectors, which represent the field in $x, y$, and $z$ directions. Then, the system is solved by a matrix solver. In this paper, we used the generalized minimal residual (GMRES) method [15].

\section{Numerical Results}

In this section we test the accuracy of the interpolated weights on tensorproduct nodes, the $\delta$-independence of the solution of the VIE, and convergence of the $p$-refinement of the Nyström collocation method.

\subsection{Accuracy of the interpolated weights on tensor-product nodes for comput- ing matrix entries}

In this subsection, the accuracy of the interpolated weights on tensorproduct on a cube is presented. The study on a sphere can be treated in a similar way.

In (46), the calculation of matrix $\mathbf{B}$ from the correction terms is straightforward; so we will focus on validating the interpolated weights in computing

matrix A. For convenience, we consider the integral of a real-valued, tensor function

$$
\frac{\cos R}{R}(\mathbf{I}-\mathbf{u} \otimes \mathbf{u})+\frac{\cos R}{R^{2}}(\mathbf{I}-3 \mathbf{u} \otimes \mathbf{u})+\frac{\cos R}{R^{3}}(\mathbf{I}-3 \mathbf{u} \otimes \mathbf{u}),
$$

which is similar to the Green's function in (44) in the domain $\Omega \backslash V_{\delta}$. Without loss of generality, we take $\Omega=[-1,1]^{3}$ and $\mathbf{r}_{j}$ as the 27 points constructed

from the tensor product of the Gauss points of order 3 in $[-1,1]$. Thus, we have $j=m=1,2, \ldots, 27$ as in (49) and the integral can be written as

$$
\mathbf{G}_{j} \approx \sum_{m=1}^{27} \cos \left(\left|\mathbf{r}_{m}-\mathbf{r}_{j}\right|\right)\left[\left(\omega_{j, m}+\bar{\omega}_{j, m}+\tilde{\omega}_{j, m}\right) \overline{\mathbf{I}}-\Lambda_{j, m}-3 \bar{\Lambda}_{j, m}-3 \tilde{\Lambda}_{j, m}\right]
$$

which is a $3 \times 3$ matrix depending on $\mathbf{r}_{j}$. 
Table 1

Convergence of the integral when the singularity is at center. $g_{11}=g_{22}=g_{33}$ and $g_{12}=g_{13}=\underline{g_{23}=0 .}$

\begin{tabular}{l|l|l|l|l}
\hline \hline & $\delta=0.1$ & $\delta=0.05$ & $\delta=0.025$ & $\delta=0.0125$ \\
\hline$g_{11}$ & 3.985701 & 4.017024 & 4.024872 & 4.026835 \\
error & $-4.1784 \mathrm{E}-2$ & $-1.0461 \mathrm{E}-2$ & $-2.613 \mathrm{E}-3$ & $-6.5 \mathrm{E}-4$ \\
order & - & 2 & 2 & 2 \\
\hline \hline
\end{tabular}

Table 2

Convergence of the integral when singularity is at a corner. Reference solution $g_{11}=$ $g_{22}=g_{33}=0.982526$ and $g_{12}=g_{13}=g_{23}=-0.998097$.

\begin{tabular}{l|l|l|l|l}
\hline \hline & $\delta=0.1$ & $\delta=0.05$ & $\delta=0.025$ & $\delta=0.0125$ \\
\hline$g_{11}$ & 0.940714 & 0.972063 & 0.979913 & 0.981876 \\
error & $-2.80425 \mathrm{E}-1$ & $-7.3424 \mathrm{E}-2$ & $-1.8102 \mathrm{E}-2$ & $-3.983 \mathrm{E}-3$ \\
order & - & 1.93 & 2 & 2.1 \\
\hline \hline$g_{12}$ & -0.998084 & -0.998094 & -0.998097 & -0.998097 \\
error & $1.3 \mathrm{E}-5$ & $3.0 \mathrm{E}-6$ & 0 & 0 \\
order & - & 2.1 & - & - \\
\hline \hline
\end{tabular}

For each $\mathbf{G}_{j}$, we use the direct brute force method introduced in [21] to obtain the reference value of the matrix entries with a small $\delta=10^{-3}$. Then we calculate the integral using the interpolated weights as in (53) with different values of $\delta$. According to the previous analysis, the error should decay on the order $O\left(\delta^{2}\right)$.

We classify the 27 sets of weights into four categories, based on the position of the singularity $\mathbf{r}_{j}$, as being near the corner, edge, face, and center of the cube. The matrix $\mathbf{G}_{j}$ is symmetric, so we only check the three diagonal entries $\left(g_{11}, g_{22}\right.$, and $\left.g_{33}\right)$ and the three upper triangular entries $\left(g_{12}, g_{13}\right.$, and $\left.g_{23}\right)$.

Table 1 presents the numerical results when the singularity $\mathbf{r}_{j}$ is located in the center of the cube, in which case $g_{11}=g_{22}=g_{33}$ and the off-diagonal entries are all zeros. The reference solution is $g_{11}=4.027477$ while the $g_{11}$ computed with $\delta=0.1,0.05,0.025$, and 0.0125 are $3.985701,4.017024,4.024872$, and 4.026835 , respectively.

In a similar fashion, Tables $2-4$ show the accuracy when the singularity is located near the corner, edge, and face of the cube, respectively. When compared to the reference values, the expected $O\left(\delta^{2}\right)$ behavior is confirmed. 
Table 3

Convergence of the integral when singularity is at an edge. Reference solutions

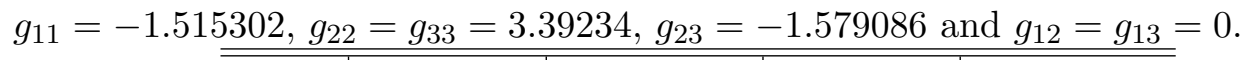

\begin{tabular}{l|l|l|l|l}
\hline \hline & $\delta=0.1$ & $\delta=0.05$ & $\delta=0.025$ & $\delta=0.0125$ \\
\hline$g_{11}$ & -1.559532 & -1.526351 & -1.518059 & -1.515987 \\
error & $-4.423 \mathrm{E}-2$ & $1.1049 \mathrm{E}-2$ & $2.757 \mathrm{E}-3$ & $-6.85 \mathrm{E}-4$ \\
order & - & 2 & 2 & 2 \\
\hline \hline$g_{22}$ & 3.35175 & 3.38217 & 3.389798 & 3.391707 \\
error & $-4.059 \mathrm{E}-2$ & $-1.1017 \mathrm{E}-2$ & $2.542 \mathrm{E}-3$ & $-6.33 \mathrm{E}-4$ \\
order & - & 1.9 & 2.1 & 2 \\
\hline \hline$g_{23}$ & -1.579072 & -1.579082 & -1.579085 & -1.579085 \\
error & $1.4 \mathrm{E}-5$ & $4.0 \mathrm{E}-6$ & $1.0 \mathrm{E}-6$ & $1.0 \mathrm{E}-6$ \\
order & - & 1.8 & 2 & 0 \\
\hline \hline
\end{tabular}

Table 4

Convergence of the integral when singularity is at a face. Reference solutions $g_{11}=$ $0.877428, g_{22}=0, g_{33}=6.494784$, and $g_{12}=g_{13}=g_{23}=0$.

\begin{tabular}{l|l|l|l|l}
\hline \hline & $\delta=0.1$ & $\delta=0.05$ & $\delta=0.025$ & $\delta=0.0125$ \\
\hline$g_{11}$ & 0.83442 & 0.866672 & 0.874742 & 0.87676 \\
error & $-4.3008 \mathrm{E}-2$ & $-1.10756 \mathrm{E}-2$ & $-2.686 \mathrm{E}-3$ & $-6.68 \mathrm{E}-4$ \\
order & - & 1.9 & 2 & 2 \\
\hline \hline$g_{33}$ & 6.455419 & 6.484909 & 6.492315 & 6.49417 \\
error & $-3.9365 \mathrm{E}-2$ & $-9.875 \mathrm{E}-3$ & $-2.469 \mathrm{E}-3$ & $-6.14 \mathrm{E}-4$ \\
order & - & 2 & 2 & 2 \\
\hline \hline
\end{tabular}

\subsection{Exclusion volume $\delta$-independence of the VIE solution}

Equation (30) provides a formulation with which the solution of the VIE will be independent of the choice of the exclusion volume size $\delta$. In the following tests, we take $\mu=1, \Delta \epsilon=4, \omega=1$ and solve the VIE. The computational domain is taken as $[-\pi / 2, \pi / 2]^{3}$, while the incident wave is

$$
\mathbf{E}_{x}^{\mathrm{inc}}=e^{i k(-y+0.5 z)}, \quad \mathbf{E}_{y}^{\mathrm{inc}}=\mathbf{E}_{z}^{\mathrm{inc}}=0 .
$$

We first check the $\delta$-independence of the matrix entries in (51). Fig. 1 displays the differences of one row of entries in the matrix $\mathbf{V}$ between the choices of $\delta=0.1$ and $\delta=0.001$, in which the solid lines are for the entries from a diagonal block $\left(V_{x x}\right)$ and dashed lines are for the entries from an off-diagonal 


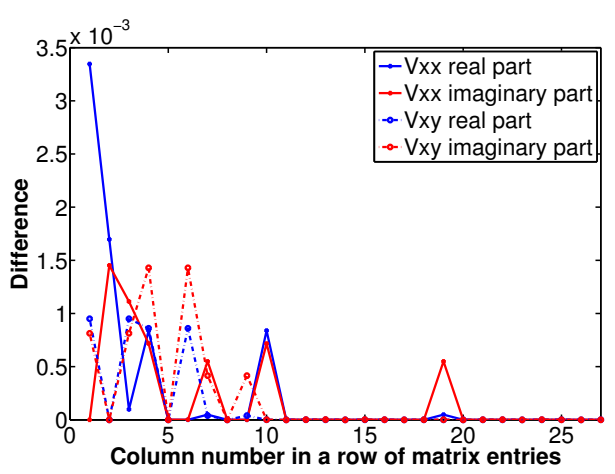

(a)

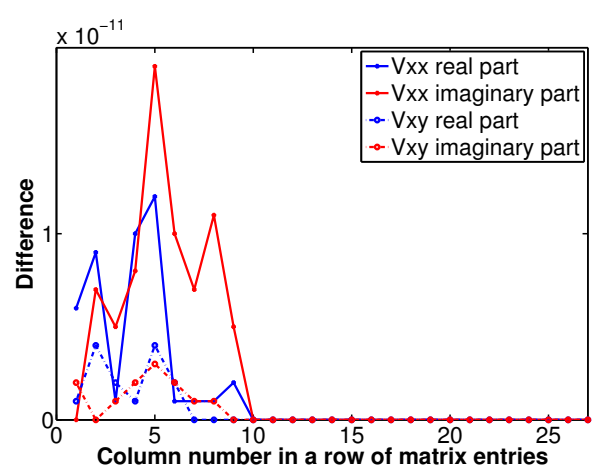

(b)

Fig. 1. Differences of matrix entries with $\delta=0.1$ and $\delta=0.001$. (a): without correction terms; (b) with correction terms.

block $\left(V_{x y}\right)$. The blue curves are for real while red curves are for imaginary parts. From Fig. 1(a) we can see that the differences between entries in the corresponding positions can be as large as $3.0 \times 10^{-3}$ when the correction terms are not included. In contrast, the corresponding differences are reduced to below $2 \times 10^{-11}$ when the corrections are included. Hence, the matrix entries are $\delta$-independent when the correction terms are included.

Next we check the $\delta$-dependence of the overall solution of the VIE. The solution of VIE with a very small $\delta=0.001$ is chosen as the reference solution. Then, the numerical solutions are computed with $\delta=0.1,0.05,0.025$, and 0.0125 and compared with the reference solution. The differences are measured in the $L^{\infty}$ norm for the three components $E_{x}, E_{y}, E_{z}$ and they are listed without and with the correction terms in Tables 5 and 6 , respectively.

Table 5

Comparison of solutions of the VIE without the correction terms

\begin{tabular}{l|l|l|l|l}
\hline \hline & $\delta=0.1$ & $\delta=0.05$ & $\delta=0.025$ & $\delta=0.0125$ \\
\hline$\left\|E_{x}-E_{x}^{\mathrm{ref}}\right\|_{L^{\infty}}$ & $3.360 \mathrm{E}-3$ & $8.264 \mathrm{E}-4$ & $2.044 \mathrm{E}-4$ & $5.039 \mathrm{E}-5$ \\
$\left\|E_{y}-E_{y}^{\mathrm{ref}}\right\|_{L^{\infty}}$ & $1.476 \mathrm{E}-3$ & $3.696 \mathrm{E}-4$ & $9.258 \mathrm{E}-5$ & $2.358 \mathrm{E}-5$ \\
$\left\|E_{z}-E_{z}^{\mathrm{ref}}\right\|_{L^{\infty}}$ & $2.533 \mathrm{E}-3$ & $6.387 \mathrm{E}-4$ & $1.597 \mathrm{E}-4$ & $3.969 \mathrm{E}-5$ \\
\hline \hline
\end{tabular}

Table 6

Comparison of solutions of the VIE with the correction terms

\begin{tabular}{l|l|l|l|l}
\hline \hline & $\delta=0.1$ & $\delta=0.05$ & $\delta=0.025$ & $\delta=0.0125$ \\
\hline$\left\|E_{x}-E_{x}^{\mathrm{ref}}\right\|_{L^{\infty}}$ & $8.0 \mathrm{E}-12$ & $1.0 \mathrm{E}-12$ & 0 & 0 \\
$\left\|E_{y}-E_{y}^{\mathrm{ref}}\right\|_{L^{\infty}}$ & $2.0 \mathrm{E}-12$ & $1.0 \mathrm{E}-12$ & 0 & 0 \\
$\left\|E_{z}-E_{z}^{\mathrm{ref}}\right\|_{L^{\infty}}$ & $1.0 \mathrm{E}-12$ & 0 & 0 & 0 \\
\hline \hline
\end{tabular}


From Table 5 it can be seen that without the correction terms, the solution of VIE has an obvious dependence on the choice of $\delta$ and the differences decreases in the order of $O\left(\delta^{2}\right)$, while the solution is indeed $\delta$-independent when the correction terms are included, as shown in Table 6.

\section{3 -convergence of the VIE solution}

For numerical convergence study, we can either discretize the computational domain into smaller elements (increasing the number $N, h$-refinement) or use higher order polynomial basis (increasing the number $p, p$-refinement) in computing the integral in the VIE. In the current work we focus on the latter since the emphasis is on the accurate calculation of the CPV of the integral. We will check the solution of VIE on a sphere and a cube. To verify the rate of convergence, we consider the linear relation between the $\log _{10}$ of the energy error and $p$, the effective number of collocation points in one direction, i.e.

$$
\log _{10}(\text { Error })=\alpha p+\beta
$$

where

$$
\text { Error }=\left\|\mathbf{E}^{p}-\mathbf{E}^{\mathrm{ref}}\right\|_{L^{2}(\Omega)},
$$

and $\mathbf{E}^{p}$ is the solution computed with $p$ collocation points in one direction, $\mathbf{E}^{\text {ref }}$ is the reference solution, and $\alpha$ and $\beta$ are fitting parameters. In previous sections we know that the solution does not depend on the choice of parameter $\delta$, so we take $\delta=0.001$ for the rest of calculations.

- Case 1: Solution of the VIE for the MIE scattering of a sphere

The analytic solution of the VIE in a sphere is given by the Mie series [11]. For an incident wave

$$
\mathbf{E}^{\mathrm{inc}}=\mathbf{i}_{x} e^{i k z},
$$

where $\mathbf{i}_{x}$ is the unit vector along $x$-direction in the Cartesian coordinates, the exact solution of electromagnetic fields inside a sphere can be expressed as the following series, in unit vectors of spherical coordinates,

$$
\mathbf{E}(r, \theta, \phi)=\sum_{n=1}^{\infty} \frac{i^{n}(2 n+1)}{n(n+1)}\left(c_{n} \mathbf{M}_{o 1 n}^{(1)}-i d_{n} \mathbf{N}_{e 1 n}^{(1)}\right),
$$

where the coefficients are

$$
\begin{aligned}
c_{n} & =\frac{j_{n}(k a)\left[k a h_{n}^{(1)}(k a)\right]^{\prime}-h_{n}^{(1)}(k a)\left[k a j_{n}(k a)\right]^{\prime}}{j_{n}(m k a)\left[k a h_{n}^{(1)}(k a)\right]^{\prime}-h_{n}^{(1)}(k a)\left[m k a j_{n}(m k a)\right]^{\prime}} ; \\
d_{n} & =\frac{m j_{n}(k a)\left[k a h_{n}^{(1)}(k a)\right]^{\prime}-m h_{n}^{(1)}(k a)\left[k a j_{n}(k a)\right]^{\prime}}{m^{2} j_{n}(m k a)\left[k a h_{n}^{(1)}(k a)\right]^{\prime}-h_{n}^{(1)}(x)\left[m k a j_{n}(m k a)\right]^{\prime}} .
\end{aligned}
$$


and the vector special harmonics are defined by

$$
\begin{gathered}
\mathbf{M}_{\mathbf{o 1 n}}^{(\mathbf{1})}=\left(\begin{array}{c}
0 \\
\cos \phi \cdot \pi_{n}(\cos \theta) j_{n}(m k r) \\
-\sin \phi \cdot \tau_{n}(\cos \theta) j_{n}(m k r)
\end{array}\right), \\
\mathbf{N}_{\mathbf{e} 1 \mathbf{n}}^{(\mathbf{1})}=\left(\begin{array}{c}
n(n+1) \cos \phi \cdot \sin \theta \cdot \pi_{n}(\cos \theta) \frac{j_{n}(m k r)}{m k r} \\
\cos \phi \cdot \tau_{n}(\cos \theta) \frac{\left[m k r j_{n}(m k r)\right]^{\prime}}{m k r} \\
-\sin \phi \cdot \pi_{n}(\cos \theta) \frac{\left[m k r j_{n}(m k r)\right]^{\prime}}{m k r}
\end{array}\right) .
\end{gathered}
$$

In the above formulas, $m$ is the refractive index of the sphere relative to the ambient medium, $a$ the radius of the sphere and $k$ is the wave number of the ambient medium. The functions $j_{n}(z)$ and $h_{n}^{(1)}(z)$ are spherical Bessel functions of first and third kind, respectively, and their derivatives have the relations [1]

$$
\left[z j_{n}(z)\right]^{\prime}=z j_{n-1}(z)-n j_{n}(z) ;\left[z h_{n}^{(1)}(z)\right]^{\prime}=z h_{n-1}^{(1)}(z)-n h_{n}^{(1)}(z) .
$$

$\pi_{n}(\cos \theta)$ and $\tau_{n}(\cos \theta)$ have the relations

$$
\pi_{n}=\frac{2 n-1}{n-1} \cos \theta \cdot \pi_{n-1}-\frac{n}{n-1} \pi_{n-2} ; \tau_{n}=n \cos \theta \cdot \pi_{n}-(n+1) \pi_{n-1},
$$

with

$$
\pi_{0}=0 ; \pi_{1}=1 ; \pi_{2}=3 \cos \theta ; \tau_{0}=0 ; \tau_{1}=\cos \theta ; \tau_{2}=3 \cos 2 \theta
$$

To find the interpolated quadrature weights in (43), Lagrange interpolation is used along the $r$-direction and $m_{r}$ Gauss nodes are used while Fourier interpolation is applied for the $\phi \in[0,2 \pi)$ and $\theta \in[0, \pi]$. In total $m_{\theta}+1$ and $2 m_{\phi}$ grid points (namely, the collocation points in the Nyström method) are equally distributed for $\theta$ and $\phi$, respectively. Therefore, the equivalent number of collocation points in each direction is calculated as $p=\sqrt[3]{m_{r}\left(2 m_{\phi}\left(m_{\theta}-1\right)+2\right)}$. The specific quadrature weights for these nodes can be found in [21].

Fig. 2 shows the error in terms of the equivalent collocation points in one direction. In this case, the Mie solution (55) is used as the reference solution with the parameters $a=0.2, k=1, m=\sqrt{2}$ (or $\Delta \epsilon=1$ ). From the fitted lines, it can be seen that the $\log _{10}$ (Error) decays linearly with respect to $p$. Quantitatively, we have

$$
\alpha=-0.38, \quad \beta=-1.95 \text {. }
$$

Hence, we conclude that the exponential convergence is achieved for the errors against the number $p$. Fig. 3 plots the numerical solution of the VIE in the sphere with 42 collocation points. 


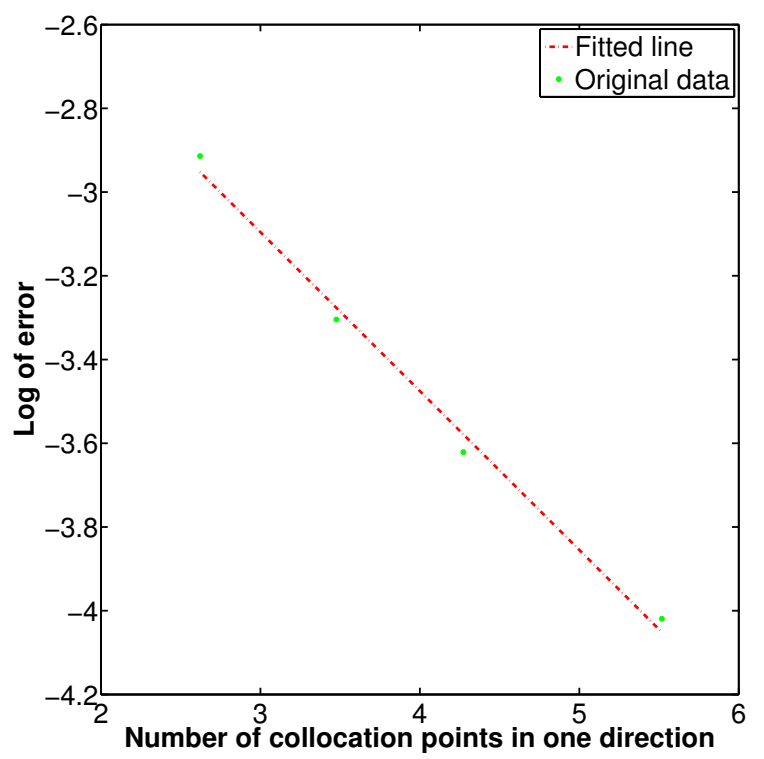

Fig. 2. $\log _{10}$ errors of solutions against number of collocation points along one direction for $\mathbf{E}$ in a sphere.

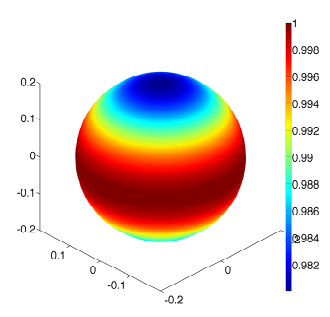

(a)

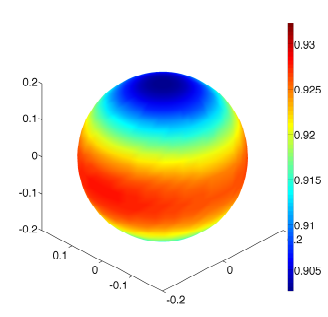

(b)

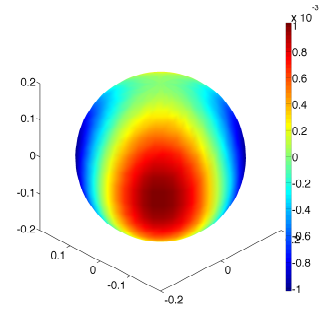

(c)

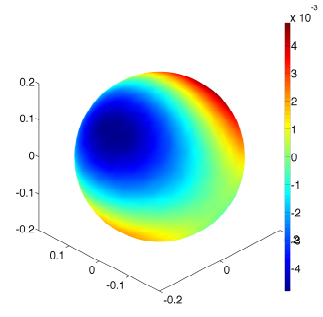

(d)

Fig. 3. Numerical solution of scattering of a sphere (radius $a=0.2$ ) with 42 collocation points. (a) incident wave; (b) $E_{x} ;$ (c) $E_{y}$, (d) $E_{z}$.

- Case 2: Solution of the VIE in a cube

In the second case, we examine the convergence of the solution in a cube. A regular Lagrange interpolation is used with Gauss nodes in each direction. As there is no exact solution, the numerical solution with collocation point $p=7$ in each direction is chosen as the reference solution $\mathbf{E}^{\text {ref }}$. The differences between the reference solution $\mathbf{E}^{\text {ref }}$ and the solutions $\mathbf{E}^{p}, p=3,4,5,6$ are computed.

Fig. 4 plots the $\log _{10}$ error against $p$, the number of collocation points along each direction, as well as the fitted line, for the solution $\mathbf{E}^{p}$. It can be seen that the $\log _{10}$ (Error) decays linearly with respect to $p$. Quantitatively, we obtain

$$
\alpha=-0.464, \quad \beta=-0.092 .
$$




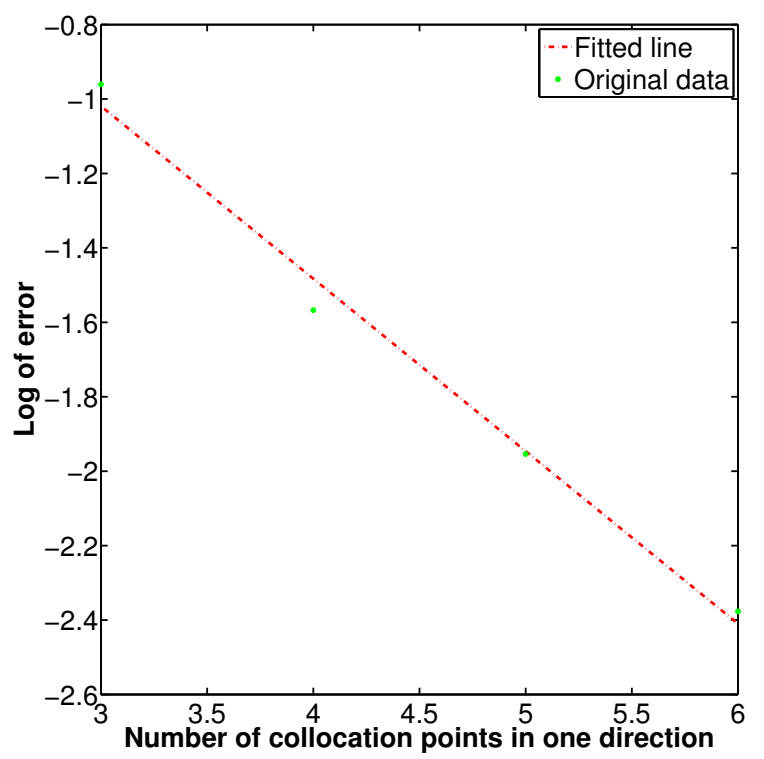

Fig. 4. $\log _{10}$ errors of solutions $\mathbf{E}^{p}$ in a cube against number of collocation points in one direction.

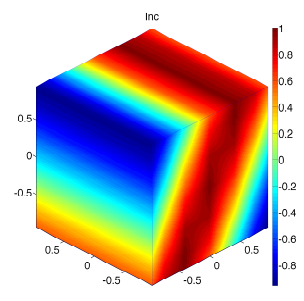

(a)

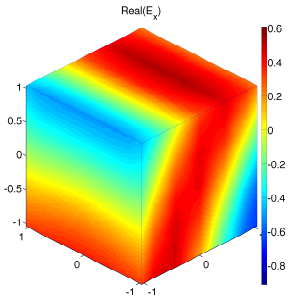

(b)

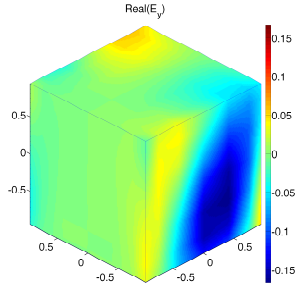

(c)

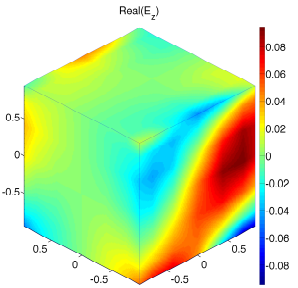

(d)

Fig. 5. Incident wave (a) and electric field (b)-(d) for a cubic scatterer with 27 collocation points.

Hence, we conclude that exponential convergence is achieved for the errors in terms of the order $p$. Fig. 5 shows the 3 -D plot of the incident wave $\mathbf{E}^{\text {inc }}=$ $\mathbf{i}_{x} e^{i k(-y+0.5 z)}$ and the resulting electric fields over the cube with 27 collocation points.

\subsection{Scattering of multiple scatterers}

Microstructures made of random scatterers, such as the rough surface of solar cell panels or meta-atoms in meta-materials, can be modeled as an array of single scatterers of fundamental shapes like cubes and spheres. In this subsection, we present the capability of our algorithm to handle multiple scatterers, either in regular or random distributions. 

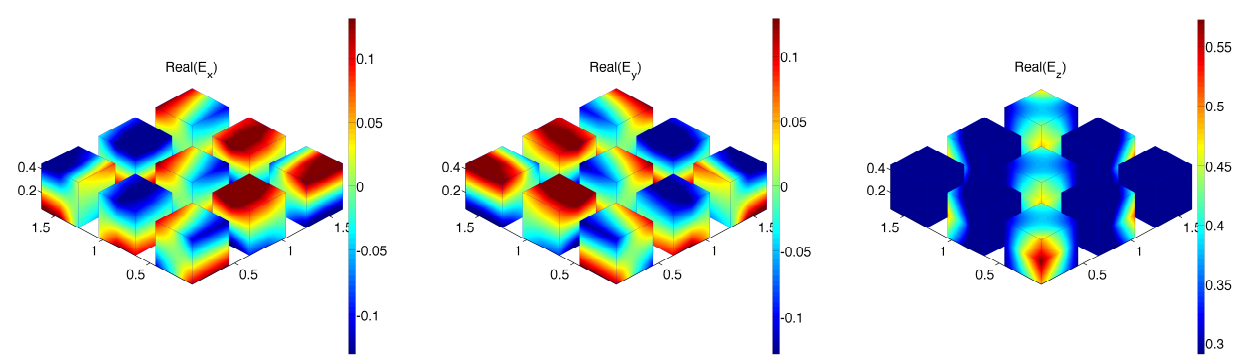

Fig. 6. Electric field ( $x$-, $y$-, and $z$-components) in a $3 \times 3$ cube array

Fig. 6 displays the electric field $\mathrm{E}_{x}, \mathrm{E}_{y}, \mathrm{E}_{z}$ in the free-space where nine cubic scatterers are present. In these tests, the incident wave is set as

$$
\mathbf{E}_{x}^{\mathrm{inc}}=\mathbf{E}_{y}^{\mathrm{inc}}=0, \quad \mathbf{E}_{z}^{\mathrm{inc}}=e^{i k(-2 x+2 y)}
$$

Each cube has a length of 0.5 and they form a $3 \times 3$ array align in the $x-y$ plane. The center of the first cube is $(0.25,0.25,0.25)$, and the remaining cubes are placed 0.1 apart from each other. The parameters are taken as $\Delta \epsilon=4$ and $\mu=1$. Here, 27 collocation points are used for each cube.

Fig. 7 displays the electric field $\mathrm{E}_{x}, \mathrm{E}_{y}, \mathrm{E}_{z}$ in the free-space where nine spherical scatterers are present. In these tests, the incident wave is set as

$$
\mathbf{E}_{x}^{\mathrm{inc}}=e^{i k z}, \quad \mathbf{E}_{y}^{\mathrm{inc}}=\mathbf{E}_{z}^{\mathrm{inc}}=0
$$

Each sphere has a radius of 1 and they form a $3 \times 3$ array align in the $x$ - $y$ plane. The center of the first sphere is $(0,0,0)$ and and the remaining sphere are placed 0.1 apart from each other. The parameters are taken as $\Delta \epsilon=1$ and $\mu=1$. Here, 42 collocation points are used for each sphere.

Due to the small number of new quadrature points needed in the Nyström VIE method, the VIE method can handle hundreds of scatterers of the fundamental shapes. The left panel of Fig. 8 show the electric field in 675 cubes of size 0.5 under the incident field (62); these non-overlapping cubes are arranged in a $15 \times 15 \times 3$ random array. Here, only 27 collocation points are needed for each cube. On the right panel of Fig. 8, the electric field in 432 spheres of radius 1 with the incident field (63) are shown. Only 42 collocation points are needed for each sphere and these non-overlapping spheres are arranged in a $12 \times 12 \times 3$ random array. In all the computations, the matrix filling is the most time consuming part. Therefore, it is computed in parallel using OpenMP. 

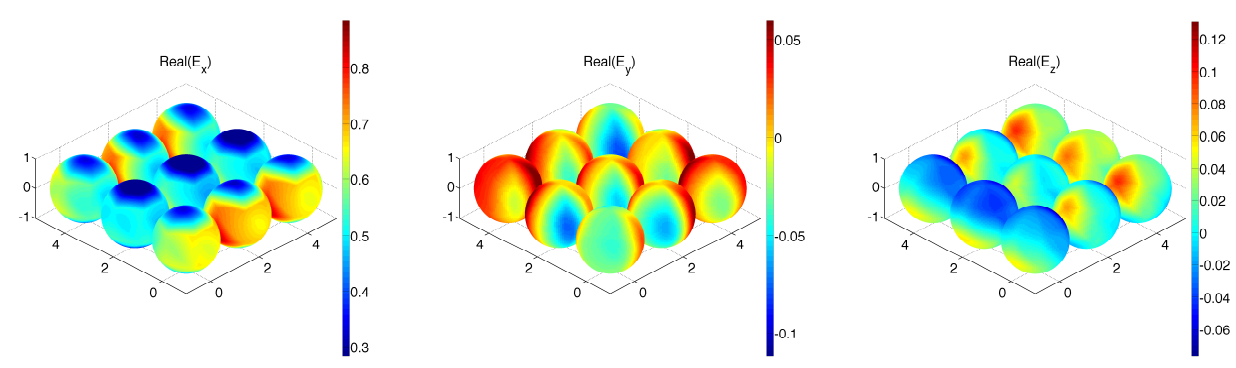

Fig. 7. Electric field $(x-, y$-, and $z$-components $)$ in a $3 \times 3$ sphere array

\section{Conclusion}

In this paper, we have developed an accurate and efficient Nyström method to simulate the scattering of multiple cubes and spheres, using the volume integral equation (VIE) for the electric field with the Cauchy principal value (CPV) of the singular dyadic Green's function. The new formulation allows the computation of the CPV using a finite size exclusion volume $V_{\delta}$ and avoiding the usual truncation errors by including missing corrections terms. As a result, the numerical solution of the VIE is shown to be $\delta$-independent. In addition, an efficient quadrature formula is employed to accurately compute the $\frac{1}{R^{3}}$ singular integration over the domain $\Omega \backslash V_{\delta}$. Together, an efficient, accurate, and $\delta$-independent Nyström collocation method for the VIE is obtained.

In various numerical tests, we demonstrated the convergence of the VIE for $p$-refinement with increasing order of basis functions (i.e. collocation points) inside a spherical and a cubic scatterer. Numerical results for the scattering of multiple scatterers of these two shapes with a small number of collocation points in each scatterer are also provided.

One of the remaining issues is the treatment of possible field singularities due to the geometric corners/edges in cubes and cylinders [18] where specially designed interpolation algorithms may be needed and corresponding interpolated quadratures will be produced. We will also study the VIE method for scattering of multiple objects embedded in layered-media for which treatment

of the singularity of layered Green's functions and fast solvers for the linear system from the Nyström method will be addressed.

\section{Acknowledgement}

The authors acknowledge the support of the US Army Office of Research (Grant No. W911NF-14-1-0297). 

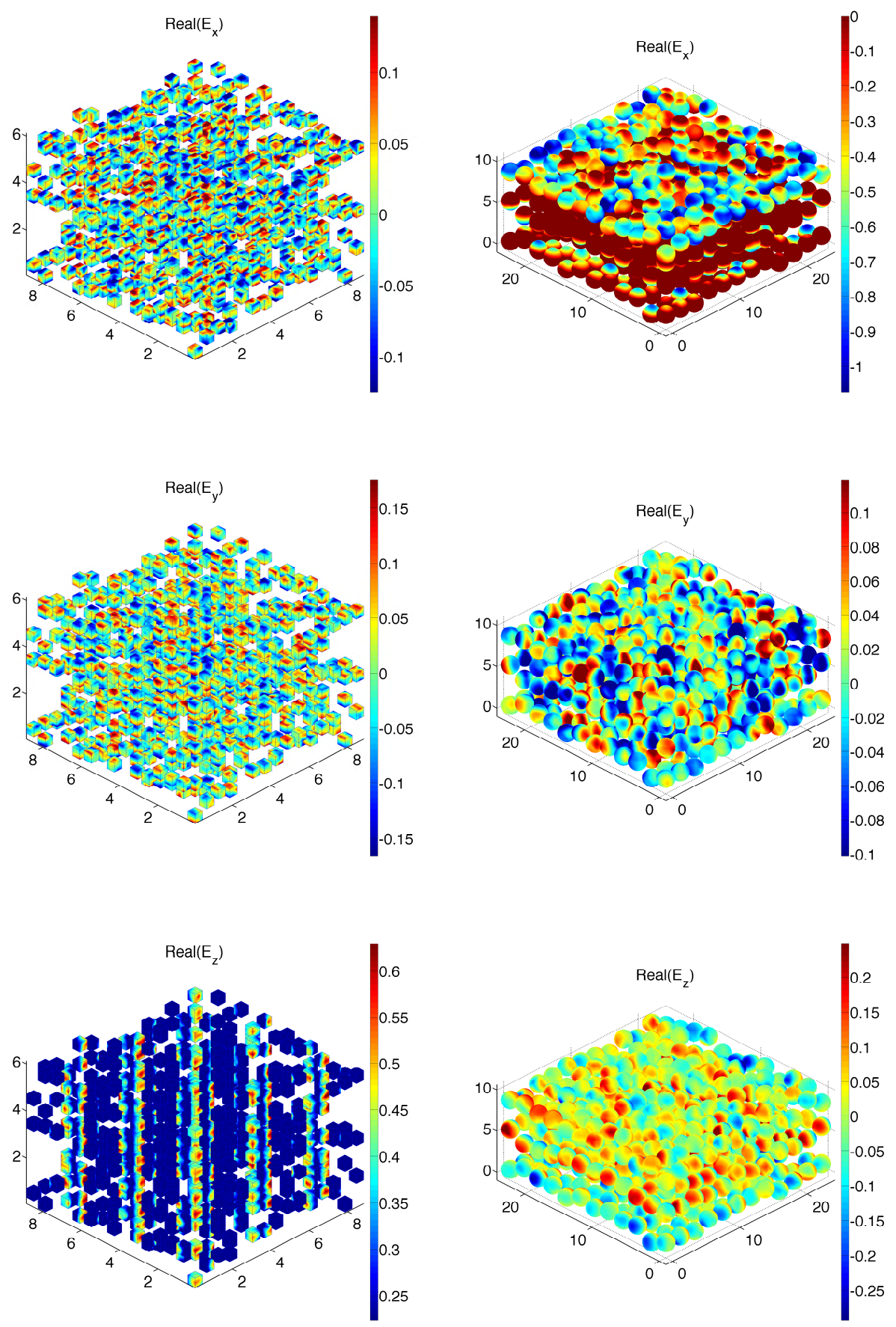

Fig. 8. (left) $E_{x}, E_{y}, E_{z}$ in a random $15 \times 15 \times 3$ cube array with 27 collocation points for each cube of size 0.5 ; (right) $E_{x}, E_{y}, E_{z}$ in a random $12 \times 12 \times 3$ sphere array with 42 collocation points for each sphere of radius 1 . 


\section{References}

[1] M. Abramowitz and I.A. Stegun. Handbook of Mathematical Functions. Dover, New York, 1970.

[2] H. Atwater. The promise of Plasmonics. Scientific American, pages 56-62, 2007.

[3] H. Atwater and A. Polman. Plasmonics for improved photovoltaic devices. Nature materials, pages 205-213, 2010.

[4] W. Cai. Computational Methods for Electromagnetic Phenomena: electrostatics in solvation, scattering, and electron transport. Cambridge University Press, 2013.

[5] J. G. Fikioris. Electromagnetic Field inside a Current-Carrying Region. Journal of Mathematical Physics, pages 1617-1620, 1965.

[6] Y.Q. Fu and X. Zhou. Plasmonic lenses: a review. Plasmonics, pages 287-310, 2010.

[7] K. Hering, D. Cialla, K. Ackermann, T. Dörefer, R. Möller, H. Schneidewind, R. Mattheis, W. Fritzsche, P. Rösch, and J. Popp. SERS: a versatile tool in chemical and biochemical diagnostics. Analytical and Bioanalytical Chemistry, pages 113-124, 2008.

[8] J.P. Kottmann and J.F. Martin, O. Accurate solution of the Volume Integral Equation for high-permittivity scatters. IEEE Transaction on Antennas and Propagation, pages 1719-1726, 2000.

[9] S.W. Lee, J. Boersma, C.L. Law, and G.A. Deschamps. Singularity in Green's function and its numerical evaluation. IEEE Transaction on Antennas and Propagation, pages 311-317, 1980.

[10] G. Liu and S.D. Gedney. High-order Nyström solution of the Volume-EFIE for TE-wave scattering. Electromagnetics, pages 1-14, 2001.

[11] G. Mie. Contribution to the optical properties of turbid media, in particular of colloidal suspensions of metals. Ann. Phys.(Leipzig), pages 377-452, 1908.

[12] J.B. Pendry. Negative refraction makes a perfect lens. Physical Review Letters, pages 3966-3969, 2000.

[13] H. Raether. Surface plasmons on smooth and rough surfaces and gratings. Springer Tracts in Modern Physics, 1988.

[14] C.V. Raman and K.S. Krishnan. A new type of secondary radiation. Nature, page 501, 1928.

[15] Y. Saad. Iterative methods for sparse linear systems. Boston, MA: PW publishing company, 1996. 
[16] J.A. Stratton. Electromagnetic Theory. New York: McGraw-Hill, 1941.

[17] M.S. Tong, Z.G. Qian, and W.C. Chew. Nyström Method Solution of Volume Integral Equations for Electromagnetic Scattering by 3D Penetrable Objects. IEEE Transaction on Antennas and Propagation, pages 1645-1652, 2010.

[18] J. van Bladel. Singular Electromagnetic Fields and Sources. Clarendon Press \& Oxford University Press, Oxford, UK, 1991.

[19] J.J.H. Wang. A Unified and Consistent View on the Singularities of the Electric Dyadic Green's Function in the Source Region. IEEE Transaction on Antennas and Propagation, pages 463-468, 1982.

[20] A.D. Yaghjian. Electric Dyadic Green's functions in the source region. Proceedings of the IEEE, pages 248-263, 1980.

[21] B. Zinser, D. Chen, and W. Cai. Quadrature Weights on Tensor-Product Nodes for Accurate Integration of Hypersingular Functions over Some Simple 3-D Geometric Shapes. submitted to Communications in Computational Physics, 2015 . 